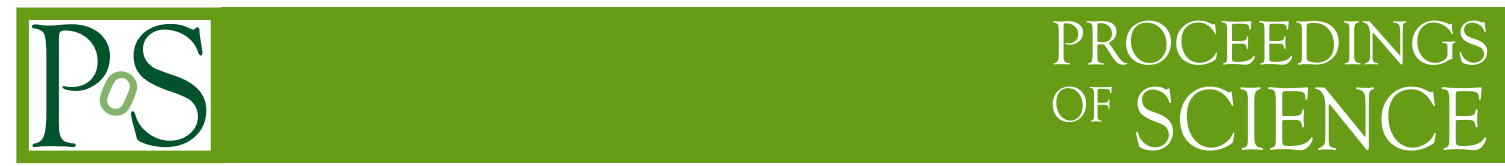

\title{
Effective action with composite fields and Clairaut-type equations
}

\section{P. Lavrov* and B. Merzlikin}

Tomsk State Pedagogical University, Russia

E-mail: Lavrovdtspu.edu.ru, merzlikindtspu.edu.ru

We consider relations existing between the effective action with composite fields and Clairaut type equations.

Corfu Summer Institute 2016 "School and Workshops on Elementary Particle Physics and Gravity" 31 August - 23 September, 2016

Corfu, Greece

${ }^{*}$ Speaker. 


\section{Introduction}

The approach to Quantum Field Theory based on concept of effective action with composite fields has been proposed by Cornwell, Jackiw and Tomboulis in 1974 [四] to improve consideration of physically important phenomena which cannot be easily seen in the perturbation series. Examples are the spontaneous symmetry violation, bound states and so on. An approximation scheme was needed that preserves some of the nonlinear features of the field theory. The original proposal dealt with the non-gauge systems. Later in [] the extension to gauge systems has been proposed. An important fact concerning the effective action with composite fields has been found in gauge systems namely its gauge independence on-shell.

The functional renormalization group approach to Quantum Field Theory proposed by Wetterich [3], 团] in the beginning of 90th presents an attempt to construct a quantization method beyond the perturbation theory by modeling expected behavior of propagators in infra-red region. This is achieved by adding regulator actions to the total action of the original theory. The regulator actions are quadratic in fields to modify the propagators. In gauge theories they violate the BRST symmetry. In its turn it leads to gauge dependence problem within the original functional renormalization group approach. The gauge dependence problem has been analyzed in details in the paper [[]]. It was proven that the effective action in gauge theories in the framework of functional renormalization group approach does depend on the gauge fixing even on-shell. Later in [目] we have found the explicit form of the effective action for an Abelian gauge model in class of linear gauges and confirmed the gauge dependence on-shell. Moreover we have proposed new formulation of the method preserving its attractive features. The formulation is based on the idea to consider regulator actions as composite fields. It leads to the known fact that the effective action with composite fields is gauge independent on-shell defining by its extremum. Therefore the study of effective action with composite fields appears to be important. It was recently realized [0] that in this study the Clairaut-type equations play very important role. Main goal of our review is to consider relations between the effective action with composite fields and Clairaut-type equations.

\section{Clairaut-type equations}

In a theory of ordinary differential equations a Clairaut equation belongs to class of non-linear first order differential equation of the form

$$
y-y^{\prime} x=\psi\left(y^{\prime}\right),
$$

where $y=y(x), y^{\prime}=d y / d x$ and $\psi=\psi(z)$ is a real function of $z$. It is well-known that the general solution to the Clairaut equation (2.1) is the family of straight line functions given by

$$
y(x)=C x+\psi(C),
$$

where $C$ is a real constant. The so-called singular solution is defined by the equation

$$
\psi^{\prime}(z)+x=0, \quad z=y^{\prime},
$$

if a solution to this equation exists in the form $z=\varphi(x)$ with a real function $\varphi$ of $x$. Then

$$
y(x)=x \varphi(x)+\psi(\varphi(x))
$$


presents the singular solution to the equation (R.D).

In connection with the Clairaut equation (R. $(\mathbb{2})$ it needs to note that there exists a lot of functions $\psi$ for which the singular solution can be constructed.

In theory of partial differential equations a Clairaut equation is defined by the first-order partial differential equation

$$
y-y_{i}^{\prime} x^{i}=\psi\left(y^{\prime}\right),
$$

which is also known as the Clairaut equation. Here $y=y(x)$ is a real function of variables $x \in \mathbf{R}^{n}$, $x=\left\{x^{1}, x^{2}, \ldots, x^{n}\right\}, \psi=\psi(z)$ is a real function of variables $z=\left\{z_{1}, z_{2}, \ldots, z_{n}\right\}$ and the notation

$$
y_{i}^{\prime}=\partial_{i} y(x) \equiv \frac{\partial y(x)}{\partial x^{i}}
$$

is used. In terms of new function $z_{i}=z_{i}(x)=y_{i}^{\prime}(x)$ the equation (2.5]) rewrites

$$
y-z_{i} x^{i}=\psi(z) .
$$

Differentiation with respect to $x^{i}$ leads to the system of differential equations

$$
\frac{\partial z_{j}}{\partial x^{i}}\left(\frac{\partial \psi}{\partial z_{j}}+x^{j}\right)=0, \quad i=1,2, \ldots, n .
$$

In the case of the Hessian matrix vanishing $H_{i j}=0$ where

$$
H_{i j}=\frac{\partial z_{j}}{\partial x^{i}}=\frac{\partial^{2} y}{\partial x^{i} \partial x^{j}}
$$

one has $z_{i}=C_{i}=$ const. Therefore the solution to the Clairaut equation is the family of linear functions

$$
y(x)=C_{i} x^{i}+\psi(C), \quad C=\left\{C_{1}, C_{2}, \ldots, C_{n}\right\} .
$$

Consider the case when

$$
\frac{\partial \psi}{\partial z_{j}}+x^{j}=0, \quad j=1,2, \ldots, n
$$

If there are any real solutions to these equations

$$
z_{j}=\varphi_{j}(x), \quad j=1,2, \ldots, n,
$$

then the singular solution to the Clairaut equation is

$$
y(x)=\varphi_{i}(x) x^{i}+\psi(\varphi(x)) .
$$

Here it should be noted that in the available scientific literature only a few examples of $\psi$ is known when the singular solutions can be constructed. 
We have found two new types of $\psi(z)$ when the singular solutions to the Clairaut - type equation admits the singular solutions:

$$
\begin{aligned}
& \text { a) } \psi(z)=-\alpha\left(z_{1} z_{2} \cdots z_{n}\right)^{\beta}, \\
& \text { b) } \psi(z)=\alpha \ln \left(1-a^{i} z_{i}\right),
\end{aligned}
$$

where $\alpha, \beta$ and $a^{i}, i=1,2, \ldots, n$ are real constants.

In the case of a) it was known ([[]]) the singular solutions when

- $n=2, \beta=1, \alpha=-1$

- $n=3, \beta=1, \alpha=-1$

- $\beta=1 /(n+1), \alpha=-(n+1)$

The case b) is most important for QFT with composite fields. We didn't find in scientific literature appropriate singular solutions of Clairaut equation in this case at all.

The singular solution in the case a) includes all known examples of choice $\psi$ listed above and has the form

$$
\text { a) } y(x)=\alpha(n \beta-1)\left(\prod_{i=1}^{n} \frac{x^{i}}{\alpha \beta}\right)^{\frac{\beta}{n \beta-1}} \text {. }
$$

In the case b) the singular solution is described with the help of constants $b_{i},(i=1,2, \ldots, n)$ as

$$
y(x)=b_{i} x^{i}-\alpha \ln \left(b_{i} x^{i}\right)-\alpha+\alpha \ln \alpha,
$$

where the constant vector $b=\left\{b_{1}, b_{2}, \ldots, b_{n}\right\}$ satisfies the condition $b_{i} a^{i}=1$. The solution ([2.17) can be considered as a new one in the theory of partial differential Clairaut equations.

We can consider the functional Clairaut-type equations. Let $\Gamma=\Gamma[F]$ be a functional of fields $F^{m}=F^{m}(x), m=1,2, \ldots, N$, which are real integrable functions of real variables $x \in \mathbf{R}^{n}$. We use the notion of functional Clairaut-type equations for the equations of the form

$$
\Gamma-\frac{\delta \Gamma}{\delta F^{m}} F^{m}=\Psi\left[\frac{\delta \Gamma}{\delta F}\right]
$$

where $\Psi=\Psi[Z]$ is a given real functional of real variables $Z_{m}=Z_{m}(x), m=1,2, \ldots, N$. Here the following notation

$$
\frac{\delta \Gamma}{\delta F^{m}} F^{m}=\int d^{n} x \frac{\delta \Gamma}{\delta F^{m}(x)} F^{m}(x)
$$

is used. The functional derivatives are defined by the rule

$$
\frac{\delta F^{m}(x)}{\delta F^{k}(y)}=\delta_{k}^{m} \delta(x-y) .
$$


In the available scientific literature we didn't find discussions concerning functional Clasirauttype equations. Nevertheless we may use the experience obtained in the case of ordinary and partial differential equations to consider some solutions to the equation (2.18)).

Let $Z_{m}(x)=\delta \Gamma / \delta F^{m}(x)$ be new unknown functions of $x$. Then

$$
\Gamma-Z_{m} F^{m}=\Psi[Z] .
$$

Varying this equation with respect to $F^{n}(y)$ we have

$$
\int d x \frac{\delta Z_{m}(x)}{\delta F^{n}(y)}\left[\frac{\delta \Psi[Z]}{\delta Z_{m}(x)}+F^{m}(x)\right]=0 .
$$

If $\delta Z_{m}(x) / \delta F^{n}(y)=0$, then

$$
\Gamma=C_{m} F^{m}+\Psi[C], \quad C_{m}=\text { const }, \quad m=1,2, \ldots, N
$$

are solutions for any set of $\left\{C_{m}\right\}$. The singular solutions (if exist) should be found from the functional system of equations

$$
\frac{\delta \Psi[Z]}{\delta Z_{m}(x)}+F^{m}(x)=0, \quad m=1,2, \ldots, N
$$

\section{Legendre transformations and Clairaut-type equations}

Let us consider a field model which is described by a non-degenerate action, $S[\phi]$, of the scalar field $\phi=\phi(x)$. The generating functional of the Green functions, $Z[J]$, and the generating functional of the connected Green functions, $W[J]$, are defined in the standard way

$$
Z[J]=\int \mathscr{D} \phi e^{i(S[\phi]+J \phi)}=e^{i W[J]},
$$

where $J=J(x)$ is usual source to $\phi$ and $J \phi=\int d x J(x) \phi(x)$. The effective action, $\Gamma=\Gamma[\Phi]$, is introduced by the Legendre transformation of $W[J]$

$$
\begin{gathered}
\Gamma[\Phi]=W[J]-J \Phi, \\
\frac{\delta W[J]}{\delta J(x)}=\Phi(x), \quad \frac{\delta \Gamma[\Phi]}{\delta \Phi(x)}=-J(x) .
\end{gathered}
$$

Eliminating the source $J$ one obtains the equation $(\Gamma=\Gamma[\Phi])$

$$
\Gamma-\frac{\delta \Gamma}{\delta \Phi} \Phi=W\left[-\frac{\delta \Gamma}{\delta \Phi}\right]
$$

which has exactly the form of functional Clairaut-type equation. The practical application requires solving the equation for $W[J]$. The standard way to find a solution to this equation is related to using the perturbation theory. In the tree approximation, $\Gamma[\Phi]=\Gamma^{(0)}[\Phi]$, it follows $\Gamma^{(0)}[\Phi]=S[\Phi]$. In the one-loop approximation, $\Gamma[\Phi]=S[\Phi]+\Gamma^{(1)}[\Phi]$, one derives

$$
\Gamma^{(1)}[\Phi]=\frac{i}{2} \operatorname{Tr} \ln S^{\prime \prime}[\Phi], \quad S^{\prime \prime}[\Phi](x, y)=\frac{\delta^{2} S[\Phi]}{\delta \Phi(x) \delta \Phi(y)}
$$

In fact, specific features of the Clairaut-type equation disappear within the perturbation expansion. 


\section{Effective action with composite fields}

Consider now an approach to QFT based on concept of composite fields. The starting point of such the approach is the generating functional of Green functions $Z[J, K]$,

$$
Z[J, K]=\int \mathscr{D} \phi e^{i(S[\phi]+J \phi+K L(\phi))}=e^{i W[J, K]},
$$

where $W=W[J, K]$ is the generating functional of connected Green functions. Here $J=J(x)$ and $K=K(x, y)$ are sources to field $\phi=\phi(x)$ and composite field $L(\phi)=L(\phi)(x, y)$, respectively, and the notation $K L(\phi)=\int d x d y K(x, y) L(\phi)(x, y)$ is used. Let $L(\phi)(x, y)$ depend quadratically on the field $\phi$

$$
L(\phi)(x, y)=\frac{1}{2} \phi(x) \phi(y) .
$$

The effective action with composite field, $\Gamma=\Gamma[\Phi, F]$, is defined by using the double Legendre transformation

$$
\begin{gathered}
\Gamma[\Phi, F]=W[J, K]-J \Phi-K\left(L(\Phi)+\frac{1}{2} F\right), \\
\frac{\delta W[J, K]}{\delta J(x)}=\Phi(x), \quad \frac{\delta W[J, K]}{\delta K(x, y)}=L(\Phi)(x, y)+\frac{1}{2} F(x, y), \\
\frac{\delta \Gamma[\Phi, F]}{\delta \Phi(x)}=-J(x)-\int d y K(x, y) \Phi(y), \quad \frac{\delta \Gamma[\Phi, F]}{\delta F(x, y)}=-\frac{1}{2} K(x, y),
\end{gathered}
$$

Eliminating the sources $J$ and $K$ one obtains the equation

$$
\Gamma-\frac{\delta \Gamma}{\delta \Phi} \Phi-\frac{\delta \Gamma}{\delta F} F=W\left[-\frac{\delta \Gamma}{\delta \Phi}+2 \frac{\delta \Gamma}{\delta F} \Phi,-2 \frac{\delta \Gamma}{\delta F}\right]-2 \frac{\delta \Gamma}{\delta F} L(\Phi) .
$$

Since the right-hand side of the last equation depends on the fields $\Phi$ not only through derivatives of functional $\Gamma=\Gamma[\Phi, F]$, the equation (4.6) does not belong to the Clairaut-type equation.

But, the one-loop approximation for the effective action with composite field, $\Gamma^{(1)}=\Gamma^{(1)}[\Phi, F]$, by itself satisfies the equation

$$
\Gamma^{(1)}-\frac{\delta \Gamma^{(1)}}{\delta F} F=\frac{i}{2} \operatorname{Tr} \ln \left(S^{\prime \prime}[\Phi]-2 \frac{\delta \Gamma^{(1)}}{\delta F}\right)
$$

being exactly the Clairaut-type with respect to field $F$ wherein the variable $\Phi$ should be considered as a parameter.

The solution to this equation has been proposed in [四]

$$
\Gamma^{(1)}[\Phi, F]=\frac{1}{2} \operatorname{Tr}\left(F S^{\prime \prime}[\Phi]\right)-\frac{i}{2} \operatorname{Tr} \ln (i F)-\frac{i}{2} \delta(0) .
$$




\section{One loop effective action with composite fields}

In real physical situation the number of fields is more then one. It needs to generalize the results obtained in [四] to the case of a field model described by a set of scalar bosonic fields $\phi^{A}(x), A=1, . ., N$, with a classical non-degenerate action $S[\phi]$. Let $L^{i}(\phi)=L^{i}(\phi)(x, y), i=1,2, \ldots, M$, be composite non-local fields,

$$
L^{i}(\phi)(x, y)=\frac{1}{2} \mathscr{A}_{A B}^{i} \phi^{A}(x) \phi^{B}(y),
$$

where $\mathscr{A}_{A B}^{i}=\mathscr{A}_{B A}^{i}$ are constants. The generating functional of Green functions, $Z[J, K]$, is given by the following path integral

$$
Z[J, K]=\int \mathscr{D} \phi e^{i\left(S[\phi]+J_{A} \phi^{A}+K_{i} L^{i}(\phi)\right)}=e^{i W[J, K]},
$$

where $K_{i}=K_{i}(x, y), i=1,2, \ldots, M$, are sources to composite fields $L^{i}(\phi)(x, y)$.

$$
J_{A} \phi^{A}=\int d x J_{A}(x) \phi^{A}(x), \quad K_{i} L^{i}(\phi)=\int d x d y K_{i}(x, y) L^{i}(\phi)(x, y) .
$$

From the definition of $Z[J, K]$ the following relations hold

$$
\frac{1}{2} \mathscr{A}_{A B}^{j} \frac{\delta^{2} Z[J, K]}{\delta J_{A}(x) \delta J_{B}(y)}=i \frac{\delta Z[J, K]}{\delta K_{j}(x, y)}, \quad j=1,2, \ldots, N,
$$

or, in terms of the functional $W[J, K]$,

$$
\frac{1}{2} \mathscr{A}_{A B}^{j}\left[-i \frac{\delta^{2} W[J, K]}{\delta J_{A}(x) \delta J_{B}(y)}+\frac{\delta W[J, K]}{\delta J_{A}(x)} \frac{\delta W[J, K]}{\delta J_{B}(y)}\right]=\frac{\delta W[J, K]}{\delta K_{j}(x, y)} .
$$

We define the average fields $\Phi^{A}(x)$ and composite fields $F^{i}(x, y)$ as follows

$$
\frac{\delta W[J, K]}{\delta J_{A}(x)}=\Phi^{A}(x), \quad \frac{\delta W[J, K]}{\delta K_{i}(x, y)}=L^{i}(\Phi)(x, y)+\frac{1}{2} F^{i}(x, y) .
$$

The effective action with composite fields, $\Gamma=\Gamma[\Phi, F]$, is defined by using the double Legendre transformation of $W[J, K]$,

$$
\Gamma[\Phi, F]=W[J, K]-J_{A} \Phi^{A}-K_{i}\left(L^{i}(\Phi)+\frac{1}{2} F^{i}\right) .
$$

One can eliminate the sources using

$$
\begin{gathered}
\frac{\delta \Gamma[\Phi, F]}{\delta \Phi^{A}(x)}=-J_{A}(x)-\int d y K_{i}(x, y) \mathscr{A}_{A B}^{i} \Phi^{B}(y), \\
\frac{\delta \Gamma[\Phi, F]}{\delta F^{i}(x, y)}=-\frac{1}{2} K_{i}(x, y) .
\end{gathered}
$$

The basic relations (5.4), (5.5) rewritten in terms of $\Gamma[\Phi, F]$ read

$$
F^{j}(x, y)-i\left(G^{-1}\right)^{A B}(x, y) \mathscr{A}_{A B}^{j}=0,
$$


where $\left(G^{-1}\right)$ is the matrix inverse to $G$,

$$
G=\left\{G_{A B}(x, y)\right\}, \quad G_{A B}(x, y)=\Gamma_{A B}^{\prime \prime}[\Phi, F](x, y)-2 \frac{\delta \Gamma[\Phi, F]}{\delta F^{i}(x, y)} \mathscr{A}_{A B}^{i},
$$

and we have used the notation

$$
\Gamma_{A B}^{\prime \prime}[\Phi, F](x, y)=\frac{\delta^{2} \Gamma[\Phi, F]}{\delta \Phi^{A}(x) \delta \Phi^{B}(y)} .
$$

In the one-loop approximation, $\Gamma[\Phi, F]=S[\Phi]+\Gamma^{(1)}[\Phi, F]$, the equation for one-loop contribution, $\Gamma^{(1)}$, to the effective action can be found in the form

$$
\Gamma^{(1)}-\frac{\delta \Gamma^{(1)}}{\delta F^{i}} F^{i}=\frac{i}{2} \operatorname{Tr} \ln \left(S_{A B}^{\prime \prime}[\Phi]-2 \frac{\delta \Gamma^{(1)}}{\delta F^{i}} \mathscr{A}_{A B}^{i}\right),
$$

being the exact functional Clairaut-type equation with respect to fields $F^{i}$ wherein the variables $\Phi^{A}$ should be considered as parameters.

The difference between this equation and the equation appearing in the case of one field $\Phi$ is the same as between the ordinary differential Clairaut equation and the partial differential Clairaut equation.

\section{Solution to the functional Clairaut-type equations}

To solve the basic equation ([.]3]) we introduce new functions $Z_{i}(x, y)$

$$
\frac{\delta \Gamma[\Phi, F]}{\delta F^{i}(x, y)}=Z_{i}(x, y)
$$

and then we have

$$
\Gamma^{(1)}=Z_{i} F^{i}+\frac{i}{2} \operatorname{Tr} \ln Q,
$$

where the matrix $Q=\left\{Q_{A B}(x, y)\right\}$ is defined as

$$
Q_{A B}(x, y)=S_{A B}^{\prime \prime}[\Phi](x, y)-2 Z_{i}(x, y) \mathscr{A}_{A B}^{i} .
$$

Varying the functional $\Gamma^{(1)}$ with respect to $F^{i}$ we obtain

$$
\delta \Gamma^{(1)}=\delta Z_{i} F^{i}+Z_{i} \delta F^{i}+\frac{i}{2} \operatorname{Tr} Q^{-1} \delta Q
$$

where $Q^{-1}$ is the inverse to $Q$,

$$
\int d z\left(Q^{-1}\right)^{A C}(x, z) Q_{C B}(z, y)=\delta_{B}^{A} \delta(x-y) .
$$

Taking into account the explicit form of $Q_{A B}$ one obtains

$$
\int d z d z^{\prime} \frac{\delta Z_{i}\left(z, z^{\prime}\right)}{\delta F^{j}(x, y)}\left[F^{i}\left(z, z^{\prime}\right)-i\left(Q^{-1}\right)^{A B}\left(z, z^{\prime}\right) \mathscr{A}_{B A}^{i}\right]=0 .
$$


Thus the equation defining non-trivial functions $Z_{i}(x, y)$ reads

$$
F^{j}(x, y)-i\left(Q^{-1}\right)^{A B}(y, x) \mathscr{A}_{B A}^{j}=0, \quad\left(Q_{A B}(x, y)=S_{A B}^{\prime \prime}[\Phi](x, y)-2 Z_{i}(x, y) \mathscr{A}_{A B}^{i}\right) .
$$

Now we introduce a set of matrices $\mathscr{B}_{j}=\left\{\mathscr{B}_{j}^{A B}\right\}$ by the relations

$$
\mathscr{A}_{A B}^{j} \mathscr{B}_{j}^{C D}=\frac{1}{2}\left(\delta_{A}^{C} \delta_{B}^{D}+\delta_{A}^{D} \delta_{B}^{C}\right) .
$$

Then we have

$$
F^{j}(x, y) \mathscr{B}_{j}^{A B}-i\left(Q^{-1}\right)^{A B}(x, y)=0 .
$$

The matrices introduced obey the properties

$$
\mathscr{B}_{j}^{A B} \mathscr{A}_{B A}^{i}=\delta_{j}^{i} \quad \text { or } \quad \operatorname{tr} \mathscr{B}_{j} \mathscr{A}^{i}=\delta_{j}^{i},
$$

which lead to the restriction on parameters $N$ and $M$

$$
\frac{1}{2} N(N+1)=M
$$

This condition has a simple sense: in a given theory with the set of $N$ fields $\phi^{A}$ there exists exactly the $(1 / 2) N(N+1)$ independent combinations of $\phi^{A} \phi^{B}$.

Then we can rewrite the equation for $Z_{i}(x, y)$ in the form

$$
Z_{i}(x, y) \mathscr{A}_{A B}^{i}=\frac{1}{2} S_{A B}^{\prime \prime}[\Phi](x, y)-\frac{i}{2}\left(F^{i} \mathscr{B}_{i}\right)_{A B}^{-1}(x, y),
$$

where

$$
\int d z\left(F^{i} \mathscr{B}_{i}\right)_{A C}^{-1}(x, z)\left(F^{j}(z, y) \mathscr{B}_{j}\right)^{C B}=\delta_{A}^{B} \delta(x-y) .
$$

It allows us to express the $Z_{i} F^{i}$ as a functional of $\Phi, F$ with the result

$$
Z_{j} F^{j}=\frac{1}{2} \operatorname{Tr}\left(\left(F^{j} \mathscr{B}_{j}\right) S^{\prime \prime}[\Phi]\right)-\frac{i}{2} \delta(0) N .
$$

Finally we find the one-loop effective action, $\Gamma^{(1)}[\Phi, F]$, in the form

$$
\Gamma^{(1)}[\Phi, F]=\frac{1}{2} \operatorname{Tr}\left(\left(F^{j} \mathscr{B}_{j}\right) S^{\prime \prime}[\Phi]\right)-\frac{i}{2} \operatorname{Tr} \ln \left(i\left(F^{j} \mathscr{B}_{j}\right)\right)-\frac{i}{2} \delta(0) N .
$$

We have studied the case with maximum number of composite fields, $M=\frac{1}{2} N(N+1)$, being quadratic in the given scalar fields $\phi^{A}, A=1, \ldots, N$. In a similar manner one can consider the situation when the number of composite fields is less the maximum one, $L^{i}(\phi)(x, y)=\frac{1}{2} \mathscr{A}_{a b}^{i} \phi^{a}(x) \phi^{b}(y), i=$ $1, \ldots, M<\frac{1}{2} N(N+1), a=1, \ldots, n<N$. Now the matrix of second derivatives of the classical action, $S_{A B}^{\prime \prime}[\Phi]$, should be presented in the block form

$$
S_{A B}^{\prime \prime}[\Phi](x, y)=\left(\begin{array}{c|c}
S_{a b}^{\prime \prime} & S_{a \beta}^{\prime \prime} \\
\hline S_{\alpha b}^{\prime \prime} & S_{\alpha \beta}^{\prime \prime}
\end{array}\right),
$$


where $\quad a, b=1, \ldots, n$ and $\alpha, \beta=n+1, \ldots, N$.

The equation for the one-loop contribution, $\Gamma^{(1)}[\Phi, F]$, to effective action takes the form

$$
\Gamma^{(1)}-\frac{\delta \Gamma^{(1)}}{\delta F^{i}} F^{i}=\frac{i}{2} \operatorname{Tr} \ln \left(\tilde{S}_{a b}^{\prime \prime}-2 \frac{\delta \Gamma^{(1)}}{\delta F^{i}} \mathscr{A}_{a b}^{i}\right)+\frac{i}{2} \operatorname{Tr} \ln S_{\alpha \beta}^{\prime \prime},
$$

where $\tilde{S}_{a b}^{\prime \prime}=S_{a b}^{\prime \prime}-S_{a \alpha}^{\prime \prime}\left(S^{\prime \prime-1}\right)^{\alpha \beta} S_{\beta b}^{\prime \prime}$. The solution reads

$$
\begin{aligned}
\Gamma^{(1)}[\Phi, F]= & \frac{1}{2} \operatorname{Tr}\left(\left(F^{j} \mathscr{B}_{j}\right)^{a b} \tilde{S}_{b c}^{\prime \prime}[\Phi]\right)-\frac{i}{2} \operatorname{Tr} \ln \left(i\left(F^{j} \mathscr{B}_{j}\right)^{a b}\right)+ \\
& +\frac{i}{2} \operatorname{Tr} \ln S_{\alpha \beta}^{\prime \prime}[\Phi]-\frac{i}{2} \delta(0) n .
\end{aligned}
$$

Here the matrixes $\mathscr{B}_{i}^{a b}$ are introduced in the same manner as in previous case but for the $\mathscr{A}_{a b}^{i}$ ones.

\section{Conclusions}

We have studied relations existing between the Legendre transformations in Quantum Field Theory and the functional differential equation for the effective action which has the form of functional Clairaut-type equation. We have found that specific features of this equation do not hold within the perturbation theory in a quantum field theory without composite operators.

Within the approach to Quantum Field Theory based on composite fields the perturbation expansion in one-loop approximation of the effective action leads exactly to a functional Clairauttype equation with a special type of the right-hand side.

Partial first-order differential equations of Clairaut-type were our preliminary step in the study of solutions to the problem. It was shown that in case when the right-hand side of the equation has the form inspired by the real situation in Quantum Field Theory with composite fields the solution to that functional Clairaut-type equation can be found with the help of algebraic manipulations only. As far as we know, the solutions found can be considered as a new result in the theory of partial first-order differential equations of Clairaut-type.

We have found an explicit solution to the functional Clairaut-type equation appearing in the Quantum Field Theory with composite fields to define one-loop contribution to the corresponding effective action.

\section{References}

[1] J. M. Cornwell, R. Jackiw, E. Tomboulis, Effective action for composite operators, Phys. Rev. D10 (1974) 2428.

[2] P. M. Lavrov, Effective action for composite fields in gauge theories, Theor. Math. Phys. 82 (1990) 282.

[3] C. Wetterich, Averege action and the renormalization group equations, Nucl. Phys. $B 352$ (1991) 529.

[4] C. Wetterich, Exact evolution equation for the effective potential, Phys. Lett. B301 (1993) 90.

[5] P. M. Lavrov, I. L. Shapiro, On the Functional Renormalization Group approach for Yang-Mills fields, JHEP 1306 (2013) 086. 
[6] P. M. Lavrov, B. S. Merzlikin, Loop expansion of the average effective action in the functional renormalization group approach, Phys. Rev. D92 (2015) 085038.

[7] P. M. Lavrov, B. S. Merzlikin, Legendre transformations and Clairaut-type equations, Phys. Lett. B756 (2016) 188.

[8] E. Kamke, Differentialgleichungen, Loesungsmethoden Und Loesungen, II Partielle Differentialgleichungen Erster Ordnung Fuer Eine Gesuchte Funktion, (Leipzig, 1959) 